International Journal on Artificial Intelligence Tools

Vol. 24, No. 6 (2015) 1599001 (7 pages)

(C) World Scientific Publishing Company

DOI: $10.1142 /$ S0218213015990018

\title{
AUTHOR INDEX Volume 24
}

Abada, L. and Aouat, S., Tabu search to solve the shape from shading ambiguity

$24(2015) 1550035$

Abdollahzade, M. and Kazemi, R., A developed local polynomial neuro-fuzzy model for nonlinear system identification

24 (2015) 1550004

Aboalsamh, H., see Hussain, M.

Adeli, H., see Siddique, N.

Adeli, H., see Siddique, N.

Adewumi, A. O. and Arasomwan, A. M., Improved particle swarm optimizer with dynamically adjusted search space and velocity limits for global optimization

Agathokleous, M., see Tsapatsoulis, N.

Agrawal, R. K., see Verma, H.

Ahmad, T., Bebis, G., Regentova, E., Nefian, A. and Fong, T., Coupling dynamic programming with machine learning for horizon line detection

Akerkar, R., Ermolayev, V. and Bassiliades, N., Editorial

Alexakos, C., see Karathanou, K.

Ammar, H. B., see Zhou, S.

Anagnostopoulos, D., see Hatzi, O.

Andreou, A. S., see Christoforou, A.

Andreou, A. S., see Papadopoulos, H.

Aouat, S., see Abada, L.

Arasomwan, A. M., see Adewumi, A. O.

Araújo, A. F. R., see Gouvêa Jr., M. M.

Arnold, P. and Rahm, E., Automatic extraction of semantic relations from Wikipedia

Assael, J.-A. M., see Kouzinopoulos, C. S.

Ayob, M., see Yassen, E. T.

Azary, S. and Savakis, A., Grassmannian spectral regression for learning and classification

Banos, V., Blanvillain, O., Kasioumis, N. and Manolopoulos, Y., A scalable approach to harvest modern weblogs

Baritakis, M., see Fafalios, P.

Bassiliades, N., see Akerkar, R.

24 (2015) 1540016

24 (2015) 1530001

24 (2015) 1530002

24 (2015) 1550017

24 (2015) 1550009

24 (2015) 1550016

24 (2015) 1540018

24 (2015) 1502002

24 (2015) 1540002

24 (2015) 1560002

24 (2015) 1450015

24 (2015) 1560005

24 (2015) 1502005

24 (2015) 1550035

24 (2015) 1550017

24 (2015) 1450013

24 (2015) 1540010

24 (2015) 1540001

24 (2015) 1550021

24 (2015) 1540015

24 (2015) 1540005

24 (2015) 1540012

24 (2015) 1502002

24 (2015) 1450015

Bassiliades, N., see Hatzi, O.

24 (2015) 1540018 
Bebis, G., see Hussain, M.

24 (2015) 1540016

Bebis, G., Editorial

Becker, B. C., see Gonzalez, A. J.

24 (2015) 1502003

BenAyed, Y., A new SVM kernel for keyword spotting using confidence measures

Blanvillain, O., see Banos, V.

Blekas, K., see Likas, A.

Brezillon, P., see Gonzalez, A. J.

Chen, Z.-Y., see Kuo, R. J.

Cho, S.-B., see Dash, C. S. K.

Christoforou, A. and Andreou, A. S., Investigating cloud adoption using influence diagrams as a decision support model

Corcho, O., see García-Silva, A.

Crampes, M., see Plantié, M.

Daneshpazhouh, A. and Sami, A., Semi-supervised outlier detection with only positive and unlabeled data based on fuzzy clustering

24 (2015) 1550020

24 (2015) 1550010

24 (2015) 1540005

24 (2015) 1502004

24 (2015) 1550020

24 (2015) 1550019

$24(2015) 1550013$

24 (2015) 1560005

24 (2015) 1540014

24 (2015) 1540006

Dash, C. S. K., Sahoo, P., Dehuri, S. and Cho, S.-B., An empirical analysis of evolved radial basis function networks and support vector machines with mixture of kernels

Dehuri, S., see Dash, C. S. K.

Delibasis, K. K., Plagianakos, V. P. and Maglogiannis, I., Estimation of robot position and orientation using a stationary fisheye camera

Della Penna, G., Intrigila, B., Magazzeni, D. and Mercorio, F., Synthesis of cost-optimal strong plans in non-deterministic domains

$24(2015) 1550003$

Dias, M. S., see Proença, P. F.

Dinh, D., see Nguyen, N. T. H.

Djouvas, C., see Tsapatsoulis, N.

Drozda, P., see Sopyla, K.

Ermolayev, V., see Akerkar, R.

Ertas, A., see Ramamoorthy, V.

Fafalios, P., Baritakis, M. and Tzitzikas, Y., Exploiting linked data for open and configurable named entity extraction

24 (2015) 1550013

24 (2015) 1550013

$24(2015) 1560004$

Fedorova, V., Gammerman, A., Nouretdinov, I. and Vovk, V., Hypergraphical conformal predictors

Fong, T., see Ahmad, T.

Gammerman, A., see Fedorova, V.

García, A., see García-Silva, A.

García-Silva, A., Jael García-Castro, L., García, A. and Corcho, O., Building domain ontologies out of folksonomies and linked data

24 (2015) 1540014

Gaspar, F., see Proença, P. F.

24 (2015) 1540017

Gonzalez, A. J., Sherwell, B., Nguyen, J., Becker, B. C., Hung, V. and Brezillon, P., A knowledge preservation and re-use tool based on context-driven reasoning

$24(2015) 1550020$ 
Goudas, T., Louizos, C., Petasis, G. and Karkaletsis, V., Argument extraction from news, blogs, and the social web

Gouvêa Jr., M. M. and Araújo, A. F. R., Evolutionary algorithm with diversity-reference adaptive control in dynamic environments

24 (2015) 1450013

Hatzi, O., Nikolaidou, M., Vrakas, D., Bassiliades, N., Anagnostopoulos, D. and Vlahavas, I., Semantically aware web service composition through AI planning

Hegenscheid, K., see Ivanovska, T.

Hung, V., see Gonzalez, A. J.

Hussain, M., Qasem, S., Bebis, G., Muhammad, G., Aboalsamh, H. and Mathkour, H., Evaluation of image forgery detection using multi-scale Weber local descriptors

Intrigila, B., see Della Penna, G.

Iosifidis, A., Tefas, A. and Pitas, I., Human action recognition based on multi-view regularized extreme learning machine

Ivanovska, T., Laqua, R., Shahid, M. L., Linsen, L., Hegenscheid, K. and Völzke, H., Automatic pharynx segmentation from MRI data for analysis of sleep related disorders

Jael García-Castro, L., see García-Silva, A.

Jarukasemratana, S. and Murata, T., Edge weight method for community detection on mixed scale-free networks

24 (2015) 1450015

24 (2015) 1550018

24 (2015) 1550020

24 (2015) 1540016

24 (2015) 1550025

$24(2015) 1540020$

Jiang, Z. and Zhan, Y.-Z., A novel diversity-based semi-supervised learning framework with related theoretical analysis

Jiang, Z., see Rao, D.

Kalles, D., see Likas, A.

Kanavos, A., Makris, C. and Theodoridis, E., Topic categorization of biomedical abstracts

Karathanou, K., Theofilatos, K., Kleftogiannis, D., Alexakos, C., Likothanassis, S., Tsakalidis, A. and Mavroudi, S., ncRNAclass: A web platform for non-coding RNA feature calculation and microRNAs and targets prediction

Karkaletsis, V., see Goudas, T.

Kasioumis, N., see Banos, V.

Kazemi, R., see Abdollahzade, M.

Kleftogiannis, D., see Karathanou, K.

Konstantinou, N., Spanos, D.-E., Kouis, D. and Mitrou, N., An approach for the incremental export of relational databases into RDF graphs

Kordestani, J. K., see Mahdaviani, M.

Kotropoulos, C., see Pliakos, K.

Kouis, D., see Konstantinou, N.

Kouzinopoulos, C. S., Assael, J.-A. M., Pyrgiotis, T. K. and Margaritis, K. G., A hybrid parallel implementation of the Aho-Corasick and Wu-Manber algorithms using NVIDIA CUDA and MPI evaluated on a biological sequence database

24 (2015) 1550018

24 (2015) 1540014

24 (2015) 1540007

24 (2015) 1550011

24 (2015) 1550002

24 (2015) 1502004

$24(2015) 1540004$

24 (2015) 1540002

24 (2015) 1540024

24 (2015) 1540005

24 (2015) 1550004

24 (2015) 1540002

24 (2015) 1540013

24 (2015) 1550023

24 (2015) 1540021

24 (2015) 1540013

24 (2015) 1540001 
Kuo, R. J., Lin, S. H. and Chen, Z.-Y., Integration of particle swarm optimization and immune genetic algorithm-based dynamic clustering for customer clustering

24 (2015) 1550019

Lagani, V., see Tsamardinos, I.

Laqua, R., see Ivanovska, T.

Le, V. Q., see Nguyen, N. T. H.

Li, K., Qian, Y., Xu, D., Zeng, J., Wang, M. and Zhang, J., On the trace of scatter matrix difference: A convex approximate discriminant analysis formulation and its mechanism research in imperial examination system

24 (2015) 1540023

24 (2015) 1550018

24 (2015) 1550024

Li, Y., Yang, Y., Wang, W. and Yang, W., An algorithm for learning the skeleton of large Bayesian network

Likas, A., Blekas, K. and Kalles, D., Editorial

Likothanassis, S., see Karathanou, K.

Lin, S. H., see Kuo, R. J.

Lin, Y., Lu, X. and Makedon, F., Approximate planning in POMDPs with weighted graph models

Linsen, L., see Ivanovska, T.

Louizos, C., see Goudas, T.

$\mathrm{Lu}, \mathrm{X}$., see Lin, Y.

Lynce, I., see Martins, R.

Magazzeni, D., see Della Penna, G.

Maglogiannis, I., see Delibasis, K. K.

Mahdaviani, M., Kordestani, J. K., Rezvanian, A. and Meybodi, M. R., LADE: Learning automata based differential evolution

Makedon, F., see Lin, Y.

Makris, C. and Tsakalidis, A., Editorial

Makris, C., see Kanavos, A.

Manic, M., see Trifunovic, M.

Manolopoulos, Y., see Banos, V.

Manolopoulos, Y., see Siddiqui, Z. F.

Manquinho, V., see Martins, R.

Margaritis, K. G., see Kouzinopoulos, C. S.

Martinez, T., see Rudolph, G.

Martins, R., Manquinho, V. and Lynce, I., Deterministic parallel MaxSAT solving

Mathkour, H., see Hussain, M.

Mavroudi, S., see Karathanou, K.

Mendez, F., see Tsapatsoulis, N.

Mercorio, F., see Della Penna, G.

Metaxas, D., see Yu, Y.

Meybodi, M. R., see Mahdaviani, M.

Misic, D., see Trifunovic, M.

Mitkas, P. A., see Vitsios, D. M.

Mitrou, N., see Konstantinou, N.

Muhammad, G., see Hussain, M.

24 (2015) 1550015

24 (2015) 1550012

24 (2015) 1502004

24 (2015) 1540002

24 (2015) 1550019

24 (2015) 1550014

24 (2015) 1550018

24 (2015) 1540024

24 (2015) 1550014

24 (2015) 1550005

24 (2015) 1550025

24 (2015) 1560004

24 (2015) 1550023

24 (2015) 1550014

24 (2015) 1502001

24 (2015) 1540004

24 (2015) 1550006

24 (2015) 1540005

24 (2015) 1540009

24 (2015) 1550005

24 (2015) 1540001

24 (2015) 1550001

24 (2015) 1550005

24 (2015) 1540016

24 (2015) 1540002

24 (2015) 1550009

24 (2015) 1550025

24 (2015) 1540019

24 (2015) 1550023

24 (2015) 1550006

24 (2015) 1540003

24 (2015) 1540013

24 (2015) 1540016 
Murata, T., see Jarukasemratana, S.

$24(2015) 1540007$

Nazri, M. Z. A., see Yassen, E. T.

24 (2015) 1550021

Nefian, A., see Ahmad, T.

Nghiem, M.-Q., see Nguyen, N. T. H.

24 (2015) 1540018

24 (2015) 1550024

Nguyen, J., see Gonzalez, A. J.

Nguyen, N. T. H., Le, V. Q., Nghiem, M.-Q. and Dinh, D.,

24 (2015) 1550020

A general approach for word reordering in

English-Vietnamese-English statistical machine translation

Nikolaidou, M., see Hatzi, O.

Nouretdinov, I., see Fedorova, V.

Ouzounis, C. A., see Vitsios, D. M.

Papadopoulos, H. and Andreou, A. S., Editorial

Peeters, R., see Zhou, S.

Petasis, G., see Goudas, T.

Pitas, I., see Iosifidis, A.

Plagianakos, V. P., see Delibasis, K. K.

Plantié, M. and Crampes, M., Visualizing and interacting with concept hierarchies

Pliakos, K. and Kotropoulos, C., Building an image annotation and tourism recommender system

Proença, P. F., Gaspar, F. and Dias, M. S., Good appearance and 3D shape descriptors for object category recognition

Psomopoulos, F. E., see Vitsios, D. M.

Pyrgiotis, T. K., see Kouzinopoulos, C. S.

Pyun, G., see Yun, U.

Qasem, S., see Hussain, M.

Qian, Y., see Li, K.

Rahm, E., see Arnold, P.

Rakhshani, A., see Tsamardinos, I.

Ramamoorthy, V., Ertas, A. and Tate, D., Assessing a new projective problem solving tool using conjoint analysis

Rao, D. and Jiang, Z., Learning planning domain descriptions in RDDL

Regentova, E., see Ahmad, T.

Rezvanian, A., see Mahdaviani, M.

Rudolph, G. and Martinez, T., Finding the real differences between learning algorithms

Sabar, N. R., see Yassen, E. T.

Sahoo, P., see Dash, C. S. K.

Sami, A., see Daneshpazhouh, A.

Santipantakis, G. and Vouros, G. A., Decomposing ontologies for the construction of distributed knowledge bases: The mONTul method

Savakis, A., see Azary, S.

Scharl, A., see Weichselbraun, A.

24 (2015) 1540015

24 (2015) 1540008

Shahid, M. L., see Ivanovska, T.

24 (2015) 1550018

Sherwell, B., see Gonzalez, A. J.

24 (2015) 1550020 
Siddique, N. and Adeli, H., Applications of harmony search algorithms in engineering

$24(2015) 1530002$

Siddique, N. and Adeli, H., Hybrid harmony search algorithms

Siddiqui, Z. F., Tiakas, E., Symeonidis, P., Spiliopoulou, M. and Manolopoulos, Y., Learning relational user profiles and recommending items as their preferences change

Smirnov, E. N., see Zhou, S.

Sopyla, K. and Drozda, P., GPU accelerated SVM with sparse sliced ELLR-T matrix format

Spanos, D.-E., see Konstantinou, N.

Spiliopoulou, M., see Siddiqui, Z. F.

Stanchev, L., Measuring the strength of the semantic relationship between words

Stojkovic, M., see Trifunovic, M.

Streiff, D., see Weichselbraun, A.

Symeonidis, P., see Siddiqui, Z. F.

Tate, D., see Ramamoorthy, V.

Tefas, A., see Iosifidis, A.

Theodoridis, E., see Kanavos, A.

Theofilatos, K., see Karathanou, K.

Tiakas, E., see Siddiqui, Z. F.

Trajanovic, M., see Trifunovic, M.

Trifunovic, M., Stojkovic, M., Misic, D., Trajanovic, M. and Manic, M., Recognizing topological analogy in semantic network

24 (2015) 1540009

24 (2015) 1560002

24 (2015) 1450012

24 (2015) 1540013

24 (2015) 1540009

24 (2015) 1540011

24 (2015) 1550006

24 (2015) 1540008

24 (2015) 1540009

24 (2015) 1550022

24 (2015) 1540020

24 (2015) 1540004

24 (2015) 1540002

24 (2015) 1540009

24 (2015) 1550006

Tsakalidis, A., see Karathanou, K.

Tsakalidis, A., see Makris, C.

Tsamardinos, I., Rakhshani, A. and Lagani, V., Performance-estimation properties of cross-validation-based protocols with simultaneous hyper-parameter optimization

Tsapatsoulis, N., Agathokleous, M., Djouvas, C. and Mendez, F., On the design of social voting recommendation applications

Tzitzikas, Y., see Fafalios, P.

Utkin, L. V. and Zhuk, Y. A., Robust classifiers using imprecise probability models and importance of classes

$24(2015) 1550006$

24 (2015) 1540002

24 (2015) 1502001

$24(2015) 1540023$

$24(2015) 1550009$

24 (2015) 1540012

$24(2015) 1550008$

Verma, H. and Agrawal, R. K., Possibilistic intuitionistic fuzzy c-means clustering algorithm for MRI brain image segmentation

$24(2015) 1550016$

Vitsios, D. M., Psomopoulos, F. E., Mitkas, P. A. and Ouzounis, C. A., Inference of pathway decomposition across multiple species through gene clustering

$24(2015) 1540003$

Vlahavas, I., see Hatzi, O.

Vouros, G. A., see Santipantakis, G.

24 (2015) 1450015

24 (2015) 1540022

24 (2015) 1560003

Vovk, V., see Fedorova, V.

24 (2015) 1560001

Vrakas, D., see Hatzi, O.

24 (2015) 1450015

Völzke, H., see Ivanovska, T.

24 (2015) 1550018 
Wang, M., see Li, K.

24 (2015) 1550015

Wang, W., see Li, Y.

24 (2015) 1550012

Weichselbraun, A., Streiff, D. and Scharl, A., Consolidating heterogeneous enterprise data for named entity linking and web intelligence

$\mathrm{Xu}$, D., see Li, K.

24 (2015) 1540008

24 (2015) 1550015

Yang, F., see Yu, Y.

24 (2015) 1540019

Yang, W., see Li, Y.

24 (2015) 1550012

Yang, Y., see Li, Y.

24 (2015) 1550012

Yassen, E. T., Ayob, M., Nazri, M. Z. A. and Sabar, N. R., A hybrid meta-heuristic algorithm for vehicle routing problem with time windows

24 (2015) 1550021

Yoon, E., see Yun, U.

Yu, Y., Zhang, S., Yang, F. and Metaxas, D., Multi-pose and occluded facial landmark localization via sparse shape representation

24 (2015) 1550007

Yun, U., Pyun, G. and Yoon, E., Efficient mining of robust closed weighted sequential patterns without information loss

Zeng, J., see Li, K.

Zhan, Y.-Z., see Jiang, Z.

Zhang, J., see Li, K.

Zhang, S., see Yu, Y.

Zhou, S., Smirnov, E. N., Peeters, R. and Ammar, H. B., Conformal region classification with instance-transfer boosting

Zhuk, Y. A., see Utkin, L. V.

24 (2015) 1540019

24 (2015) 1550007

24 (2015) 1550015

24 (2015) 1550011

24 (2015) 1550015

24 (2015) 1540019

24 (2015) 1560002

24 (2015) 1550008 